\title{
Using Sequence-Predicted Contacts to Guide Template-free Protein Structure Prediction
}

\author{
Ahmed Bin Zaman \\ Dept of Computer Science \\ George Mason University \\ azaman6@gmu.edu
}

\author{
Prasanna Venkatesh \\ Parthasarathy \\ Dept of Computer Science \\ George Mason University \\ ppartha@gmu.edu
}

\author{
Amarda Shehu* \\ Dept of Computer Science \\ George Mason University \\ amarda@gmu.edu
}

\begin{abstract}
Template-free protein structure prediction seeks three-dimensional structures that organize the serially-bonded amino acids in ways that lower interatomic energy. It is now well understood that energy functions are unreliable guides towards biologically-active structures. This realization raises questions on the proper role and utilization of energy functions. Recent work suggests employing complementary information in the form of amino-acid contacts. Here, we advance this line of work and leverage multi-objective optimization to investigate a variety of combinations of interatomic energy and contact-based scoring. Evaluation on diverse datasets demonstrates the superiority of combining contact information with energy functions in a multi-objective optimization setting for template-free protein structure prediction.
\end{abstract}

\section{CCS CONCEPTS}

- Computing methodologies $\rightarrow$ Bio-inspired approaches; • Applied computing $\rightarrow$ Molecular structural biology; Bioinformatics.

\section{KEYWORDS}

protein structure prediction; decoy sampling; contact-based scoring; multi-objective optimization.

ACM Reference Format:

Ahmed Bin Zaman, Prasanna Venkatesh Parthasarathy, and Amarda Shehu. 2019. Using Sequence-Predicted Contacts to Guide Template-free Protein Structure Prediction. In 10th ACM International Conference on Bioinformatics, Computational Biology and Health Informatics (ACM-BCB '19), September 7-10, 2019, Niagara Falls, NY, USA. ACM, New York, NY, USA, 7 pages. https://doi.org/10.1145/3307339.3342175

\section{INTRODUCTION}

Template-free protein structure prediction (PSP) has a natural formulation as an optimization problem [12], operating under physical

\footnotetext{
${ }^{*}$ Corresponding Author
}

Permission to make digital or hard copies of all or part of this work for personal or classroom use is granted without fee provided that copies are not made or distributed for profit or commercial advantage and that copies bear this notice and the full citation on the first page. Copyrights for components of this work owned by others than ACM must be honored. Abstracting with credit is permitted. To copy otherwise, or republish, to post on servers or to redistribute to lists, requires prior specific permission and/or a fee. Request permissions from permissions@acm.org.

ACM-BCB '19, September 7-10, 2019, Niagara Falls, NY, USA

(c) 2019 Association for Computing Machinery.

ACM ISBN 978-1-4503-6666-3/19/09 . \$ $\$ 15.00$

https://doi.org/10.1145/3307339.3342175 principles demonstrated in theory and experiment that the threedimensional/tertiary structures comprising the stable, long-time populated state(s) observed in the wet laboratory [2] reside in deep and broad basins of the potential energy function summing up atomic interactions [15]. Main advances in template-free PSP relate to molecular fragment replacement, sampling, and scoring.

Briefly, known native structures deposited in databases, such as the Protein Data Bank (PDB) [4], are excised into fragments. The resulting fragment configurations are organized in a library indexed by fragment amino-acid sequences. Molecular fragment replacement can be used to introduce variation in a structure as follows: an amino acid index $i$ is selected at random over $[1, l-$ $f+1$, where $l$ is the number of amino acids in the sequence, and $f$ is the fragment length. The configuration of the selected fragment is replaced with a configuration selected at random among those available for the fragment with the same or similar aminoacid sequence. This can be considered a "move" in a local search technique such as Monte Carlo (MC), and bias can be introduced in an iterative process, as in Metropolis Monte Carlo (MMC) sampling, to obtain better-scoring (lower energy) structures.

State-of-the-art methods use fragment replacement but vary in the sampling algorithms and energy/scoring functions employed; Rosetta and Quark use Simulated Annealing MMC (SA-MMC); others use EAs for enhanced sampling $[17,18]$. Scoring functions are also diverse. While their accuracy has improved, they are still a proxy or approximation of the true internal energy. Recent work investigates learning new scoring functions based on predicted pairwise distances [7] or contacts [1] (which record whether two amino acids are spatially proximal based on a characteristic distance threshold of $8 \AA$ between the beta carbon atoms).

In particular, the growth in known native structures has allowed data-driven methods to predict contacts with increasing accuracy. The top performer in CASP12 (CASP stands for "Critical Assessment of Structure Prediction") was RaptorX-Contact [21], which employs a deep residual neural network. However, assessors in CASP12 noted that turning a better contact prediction into a better structural model remains a main challenge [19]. Various approaches have been proposed $[5,9,19,24,25]$ that either completely replace the energy function with a contact-based scoring one, or add derived contacts as restraints in a new term added to the energy function.

Aggregation of terms into a pseudo-energy function may result in a search space with many sub-optimal minima. In addition, one cannot know a priori the relative importance of one scoring function over the other. Optimization research advocates for keeping scoring criteria as separate optimization objectives, which not only avoids introducing unnecessary parameterization (term weights), 
but also leads to better and diverse optima [6]. It is also unclear how many contacts are needed to improve performance, as not all are predicted with the same confidence. When evaluating contact prediction methods, it is common to consider a reduced list of the most confident $10, l / 5$, or $l / 2$ contacts, where $l$ is the number of amino acids in a sequence [19]. Various contact-based scores are devised, such as precision, recall, $\mathrm{F} 1$, and coverage.

In this paper, we advance the argument on the role of energy versus complementary information in the form of sequence-predicted contacts. Specifically, we evaluate the derived contact list of a computed structure in relation to the sequence-predicted one from RaptorX. Unlike existing work, we do not resort to aggregating energy with contact-based scoring but consider them as separate optimization objectives, making use of multi-objective and single-objective EAs to holistically evaluate the separate versus the combined role and impact of energy versus contacts in structure prediction. Evaluation on diverse datasets yields many interesting observations and advocates the superiority of combining contact information with energy functions. Our findings suggest that neither energy functions nor contact-based scoring are sufficient by themselves as guides towards native structures; instead, they each provide complementary information that together confers better performance in a multi-objective optimization setting for template-free PSP.

The rest of this paper is organized as follows. Section 2 describes the foundational algorithmic platform and the various algorithms proposed to evaluate the impact of energy versus contact-based scoring function in isolation and combination. Section 3 presents a detailed comparative evaluation on diverse datasets. Section 4 concludes the paper with a summary and discussion of future work.

\section{METHODS}

Let us first describe in some detail local iterative search for decoy sampling. The process starts with an initial structure, where backbone dihedral angle values are sampled at random, over Ramachandran maps, or set to an extended backbone chain. The current structure serves as a parent, and molecular fragment replacement yields an offspring. In a greedy version, the offspring replaces the parent only if its energy is lower. The Metropolis criterion allows the offspring to survive and replace the parent with some probability. The process continues from the current parent. Though overly simplified, this process is the foundation of decoy sampling in Rosetta, Quark, and others. Rosetta employs SA-MMC, where the Metropolis criterion changes in accordance with a temperature parameter. The energy/scoring function also varies during the search, gradually adding more constraints to obtain increasingly realistic tertiary structures. Ref. [11] provides more details on Rosetta.

\subsection{HEA: A Hybrid Evolutionary Algorithmic Template}

In this paper, we use an EA as an algorithmic template, as it has higher sampling capability than SA-MMC [16, 17, 22]. Our baseline algorithm is a hybrid, population-based, single-objective EA, which we refer to as HEA, and which we have demonstrated to have good sampling capability in comparison to decoy sampling in Rosetta [16]. In summary, an HEA combines global and local search via the concept of a population of $N$ structures that evolve towards better-fit ones. Structures (also referred to as individuals) in a population yield new structures (offspring) via applications of one or more variation operators. Molecular fragment replacement is an example of a variation operator. The initial population is constructed carefully, starting with extended backbone chains that are randomized via repeated molecular fragment replacements (with fragments of length 9). Each parent yields an offspring via one molecular fragment replacement (using fragments of length 3). Offspring are further improved via an iterative improvement operator. This operator implements local search via iterative applications of molecular fragment replacement (with fragments of length 3). Improved offspring are combined with parents, and a selection operator selects $N$ structures for survival out of the combined pool. Each structure is evaluated via the Rosetta score4 energy function (for direct comparison with Rosetta); the function that evaluates individuals is referred to as the fitness function. The $N$ fittest (lowest-energy) structures are retained in the population, kicking off another generation. The process of varying, improving, combining, and then selecting continues until a total budget of fitness/energy evaluations has been exhausted. The reader is referred to Ref. $[16,17]$ for more implementation details.

\subsection{HEA-C: Guiding a Hybrid Evolutionary Algorithm by Contact-based Scoring}

The above selection operator can be easily modified to select individuals based on contacts. In this modified single-objective EA, which we deem HEA-C (C for 'C'ontacts), a structure is evaluated as follows: Its contacts are calculated using a threshold of $8 \AA$ to record whether distances between pairs of non-bonded CB atoms are below the threshold. RaptorX-Contact is used to predict contacts from the amino-acid sequence alone. These contacts come with (confidence) probabilities. Based on experiments we have conducted over known native structures, the top 10 RaptorX-predicted contacts lead to more accurate contact-based scoring functions (data not shown in the interest of space). In the CASP contact prediction category that evaluates sequence-based predicted contacts, the known native structure is treated as the ground truth [19]. In our setting, the native structure is not known. Instead, we treat the top 10 RaptorX-predicted contacts as the ground truth. These 10 pairs of amino acids are evaluated on whether they form a contact in a computed structure that is to be evaluated in HEA-C. If the pairs are also found in contact in a computed structure, they increment the number of true positives (TPs). Otherwise, they increment the number of false negatives (FNs) (reported to be in contact by RaptorX-Contact but not found in contact in an HEA-C computed structure). Each HEA-C structure evaluated in the selection operator is then scored via $\mathrm{TP} /(\mathrm{TP}+\mathrm{FN})$; this score is known as sensitivity (or true positive rate - TPR). In HEA-C, the top $N$ structures (with highest sensitivity scores) survive in the next generation.

\subsection{MOEANS: A Multi-objective Evolutionary Algorithmic Template}

In contrast to using energy or contact-based scoring alone, one can consider joining the two by leveraging the selection operator. As described in Section 1, we make use of a non-parametric approach via multi-objective optimization, the MOEANS platform [22]. The 
selection operator in MOEANS uses concepts of non-dominated sorting and crowding distance sorting to select $N$ structures for survival. In MOEANS, the energy terms in Rosetta's score4 are decomposed into 3 groups considered as separate objectives. As hydrogen bonding is very important for the formation of native structures [20], short- and long-range hydrogen bonding are separate objectives. The third sums up the rest of the energy terms.

MOEANS uses the concept of dominance: a structure $C_{a}$ dominates another structure $C_{b}$ if each objective score of $C_{a}$ is better than the corresponding objective score of $C_{b}$. For each structure $C$, the selection operator calculates its domination count $n_{C}$ (the number of structures that dominate $C$ ) and a set $S_{C}$ (the set of structures that $C$ dominates). If no structure in the combined population of offspring and parents dominates $C, C$ has a domination count of 0 (said to be non-dominated). The selection operator uses the concept of non-dominated fronts. All the structures with $n_{C}=0$ make up the first non-dominated front $F_{1}$. To generate the subsequent non-dominated front $F_{i}$, the following process is repeated. For each structure $C$ in $F_{i-1}$, the domination count of each member in set $S_{C}$ is decremented by 1 . As a result, the structures in $S_{C}$ that have their domination count reduced to 0 constitute the non-dominated front $F_{i}$. This process continues until the total number of structures in the generated fronts equals or exceeds $N$.

The crowding distance estimates the density of structures in the population and guides the selection operator towards less-crowded regions. The crowding distance assignment technique is used to compute the average distance of a structure from others in the same non-dominated front along each objective. First, the crowding distance of each structure is initialized to 0 . Then, for each objective, structures are sorted based on their corresponding score in ascending order; infinite distance values are assigned to the structures with highest and lowest scores, so that they are always selected. For all other structures $C$, we add to their corresponding crowding distance the absolute normalized difference in scores between the two closest structures on either side of $C$. After considering all objectives, the sum of the individual distances for each objective constitutes the crowding distance of $C$. From the combined population, the next generation is selected as follows. $p$ individuals are sequentially selected from $F_{1}, F_{2}, \ldots, F_{e}$, where $p=\sum_{i \in\{1,2, \ldots, e\}}\left|F_{i}\right|$, until $\left(p+\left|F_{e+1}\right|\right)>=N$. When this process ends and $p<N$, the structures in $F_{e+1}$ are sorted in descending order of their crowding distance and the top $N-p$ individuals are selected for survival.

\subsection{MOEANS-EC and MOEANS-SLEC: Energy- and Contact-based Scoring as Optimization Objectives}

The selection operator in MOEANS can be modified to consider other objectives as follows. First, we consider the contact-based scoring function described above and the (total) score4 energy as two separate objectives. We refer to this algorithm as MOEANSEC. Second, we consider the contact-based scoring function to be the fourth objective in addition to the 3 energetic objectives in MOEANS. We refer this algorithm as MOEANS-SLEC. We note that in these multi-objective EAs, a structure dominates another if it has a lower score for each of the energy objectives but a higher contact-based score.

\subsection{Implementation Details}

The population for each EA contains $N=100$ structures. In keeping with our earlier work that evaluates how to stall premature convergence [16], not all parents compete with offspring in HEA and HEA-C. An elitism rate of $25 \%$ is employed, where only the top $25 \%$ of the parents compete with offspring. This prevents a few fittest parents from taking over the population, effectively providing enough time for suboptimal structures to improve and contribute "genetic material" over generations. In multi-objective EAs, the diversity of objectives is sufficient to stall premature convergence. In these EAs, all parents can compete with offspring (elitism rate of $100 \%$ ). All EAs are run 5 times on each target protein's amino-acid sequence to account for their stochasticity. Each run has a budget of 10, 000, 000 fitness evaluations. The algorithms are implemented in Python, and they interface with PyRosetta and Biopython libraries.

\section{RESULTS}

\subsection{Experimental Setting}

Testing Datasets: We carry out comparative evaluations on two datasets. The first is a benchmark dataset of 20 target proteins of varying lengths (ranging from 53 to 146 amino acids) and folds ( $\alpha$, $\beta, \alpha+\beta$, and coil). This dataset was introduced in [14] and then complemented with more targets in later work [17, 22, 25]. The second dataset consists of 10 hard target domains from CASP12 and CASP13.

Comparative Evaluation Setting: HEA, HEA-C, MOEANS, MOEANS-EC, and MOEANS-SLEC, are compared to the Rosetta decoy sampling algorithm, which represents state-of-the-art, energyguided algorithms, and SCDE, a recent differential evolution algorithm [25] that does not make use of interatomic energy but penalizes computed structures by how much they deviate in their content of secondary structure elements and contacts based on predictions from sequence. To ensure a fair comparison, we have run Rosetta with a total budget of $54 M$ energy evaluations per target. Since SCDE is not available, reported results are those published in [25]; these are obtained with 30 runs of SCDE, where each run exhausts a budget of $9 \mathrm{M}$ evaluations.

Performance Metrics: Runs (per target) for each algorithm under comparison are combined, and performance is summarized by the lowest energy (score4) reached and the closest proximity to the known native structure. The first provides information on exploration capability. The second provides information on the ability to get to near-native regions of the space. Proximity to a given native structure is measured via lowest least root-mean-squared-deviation (IRMSD) [13] and GDT_TS [23]. IRMSD is a dissimilarity score and measures the Euclidean distance averaged over the atoms under comparison after the two structures under comparison are superimposed to remove differences due to rigid-body motions in 3D (rotations and translations). GDT_TS is a standard similarity score used in CASP and stands for 'Global Distance Test-Total Score' [23]. GDT_TS calculates the percentage of atoms within 2, 4, 6, $8 \AA$ and reports an average value in $[0,1]$ (which can be also interpreted as a percentage). In all comparisons, we consider achieving the same score for a target by two algorithms as a win for both.

Statistical Significance: The comparative evaluation is further strengthened by two exact statistical significance tests, Fisher's [8] 
and Barnard's [3]. Fisher's conditional exact test is used widely for statistical significance; Barnard's unconditional test is generally considered stronger for $2 \times 2$ contingency matrices.

\subsection{Evaluation on Benchmark Dataset}

We relate the comparison of HEA, HEA-C, MOEANS, MOEANSEC, MOEANS-SLEC, and Rosetta on each target of the benchmark dataset in terms of the lowest Rosetta score 4 and lowest IRMSD to the known native structure reached by each algorithm in Fig. 1. While no energy values are reported for SCDE in [25], lowest IRMSD values are reported for 15/20 target and are added in Fig. 1.
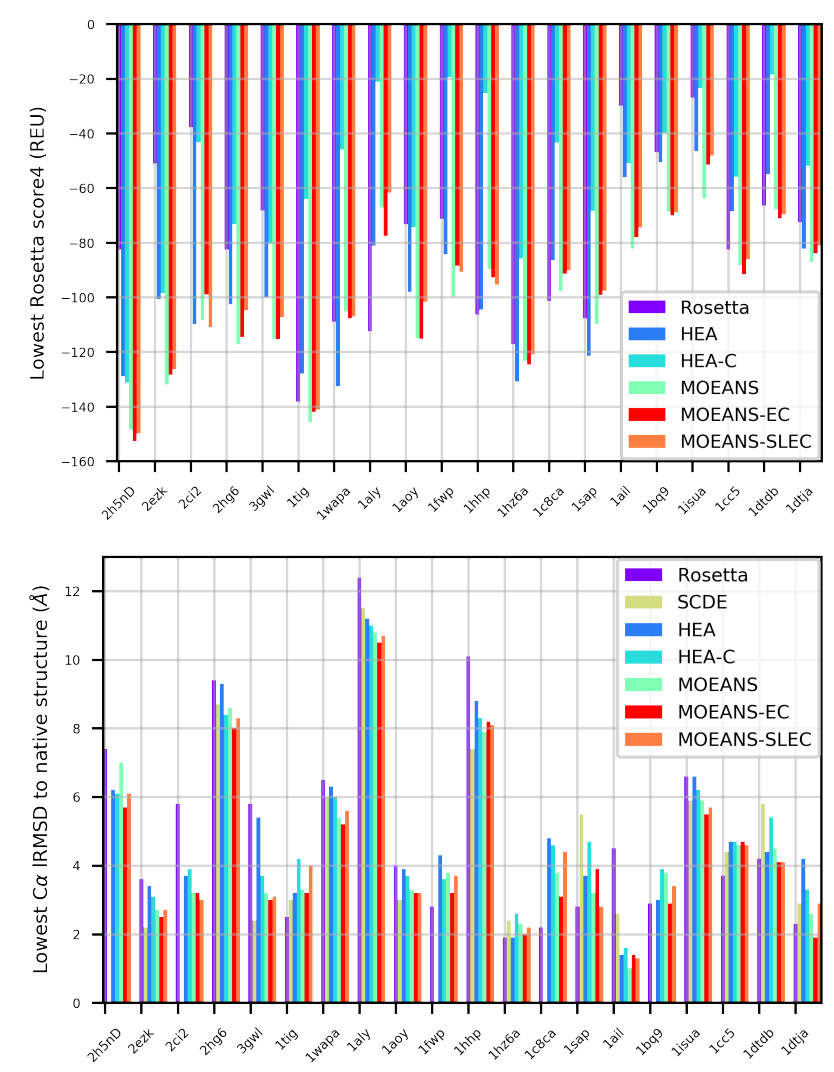

Figure 1: Comparison of (top panel) the lowest score4 (in Rosetta Energy Units - REUs) and (bottom panel) the lowest IRMSD (measured in $\AA$ ) obtained by each algorithm on each of the 20 benchmark targets.

Several observations can be drawn from the energy results related in Fig. 1. HEA-C achieves higher energy than all other algorithms. This is expected, as the selection operator in HEA-C uses only the contact-based score. MOEANS-SLEC, which implements multi-objective optimization with objectives being contact-based score, short-term hydrogen bonding, long-term hydrogen bonding, and the rest of score 4 terms as its fourth objective, reaches lower energy than Rosetta in 15/20 targets, lower energy than HEA in $14 / 20$ targets, lower energy than HEA-C in 20/20 targets, lower energy than MOEANS in only $4 / 20$ targets, and lower-energy than MOEANS-EC in only 3/20 targets. Table 1(a) which evaluates the 1-sided statistical significance tests of the performance of MOEANSSLEC over Rosetta, HEA, and HEA-C, shows that the improvements of MOEANS-SLEC over these three algorithms are statistically significant for both Fisher's and Barnard's tests ( $p$-values $<0.05$ ). This indicates that the multi-objective setting that considers contact score affords a higher exploration capability over single-objective settings, such as Rosetta, HEA, and HEA-C.

MOEANS-SLEC does not improve over MOEANS and MOEANS$\mathrm{EC}$, indicating that breaking into many objectives does not provide an advantage. MOEANS-EC, where Rosetta score4 and the contactbased score are two separate optimization objectives, reaches lower energy than Rosetta in 15/20 targets, lower energy than HEA in $14 / 20$ targets, lower energy than HEA-C in 20/20 targets, and lower energy than MOEANS-SLEC in 17/20 targets. Table 2(a) confirms that these improvements are statistically significant for both Fisher's and Barnard's tests. A tie between MOEANS-EC and MOEANS (each achieves lower energy than the other in 10/20 targets) indicates that guiding additionally by contact-based scoring does not hamper the exploration of the score4 energy surface.

Several observations can be drawn from the IRMSD results related in Fig. 1. HEA-C achieves higher IRMSD than all other algorithms except for HEA, where it wins on 13/20 targets. This is informative, considering that HEA-C performs worse than HEA and the other algorithms energy-wise. It confirms that energy guidance is not reliable, which was the motivation for the work in this paper. It also shows that selection by contact-based scoring improves proximity to the native structure over selection by energy alone. In addition, the results in Fig. 1 show that MOEANS-SLEC outperforms (reaching lower IRMSD) Rosetta in 13/20 targets, SCDE in 9/15 targets, MOEANS in 14/20 targets, HEA in 17/20 targets, and HEA-C in 19/20 targets. Table 1(b) confirms that the improvements of MOEANS-SLEC over MOEANS, HEA, and HEA-C are statistically significant. This is interesting, considering that MOEANS-SLEC performs worse than MOEANS energy-wise. The result indicates that adding contact-based score as another optimization objective guides the exploration towards regions that, while not as low in energy, are closer to the native structure.

MOEANS-EC beats all other algorithms in reaching lower IRMSDs; it outperforms Rosetta in 14/20 targets, SCDE in 9/15 targets, MOEANS in 16/20 targets, HEA in 18/20 targets, HEA-C in 20/20 targets, and MOEANS-SLEC in 15/20 targets. Table 2(b) confirms these improvements by MOEANS-EC are statistically significant over all other algorithms except for SCDE. Taken altogether, these results suggest that while injecting too many optimization objectives may not be beneficial, considering both energy and contactbased score as optimization objectives provides both high exploration capability and better proximity to the native structure.

\subsection{Evaluation on CASP12 and CASP13 Targets}

Fig. 2 compares all algorithms (except for SCDE) on CASP targets in terms of lowest score4 energy and lowest IRMSD to the known native structure. As in the benchmark dataset, HEA-C achieves higher energy than others. MOEANS-SLEC outperforms HEA in 6/10 and HEA-C in 10/10 targets. Table 1(c) confirms that this performance of MOEANS-SLEC is statistically significant. As in the benchmark dataset, MOEANS-SLEC loses to MOEANS in 7/10 targets and to 
MOEANS-EC in 8/10 targets, but ties with Rosetta, again indicating that the high number of objectives may hamper the exploration capability. MOEANS-EC beats all other algorithms energy-wise, outperforming Rosetta in 8/10 targets, MOEANS in 7/10 targets, HEA in $8 / 10$ targets, HEA-C in 10/10 targets, and MOEANS-SLEC in $8 / 10$ targets. Table 2(c) confirms these improvements by MOEANS-EC are statistically significant over all algorithms except for MOEANS (which is very close to the $95 \%$ confidence cutoff). This agrees with the evaluation on the benchmark dataset and confirms the high exploration capability of MOEANS-EC.
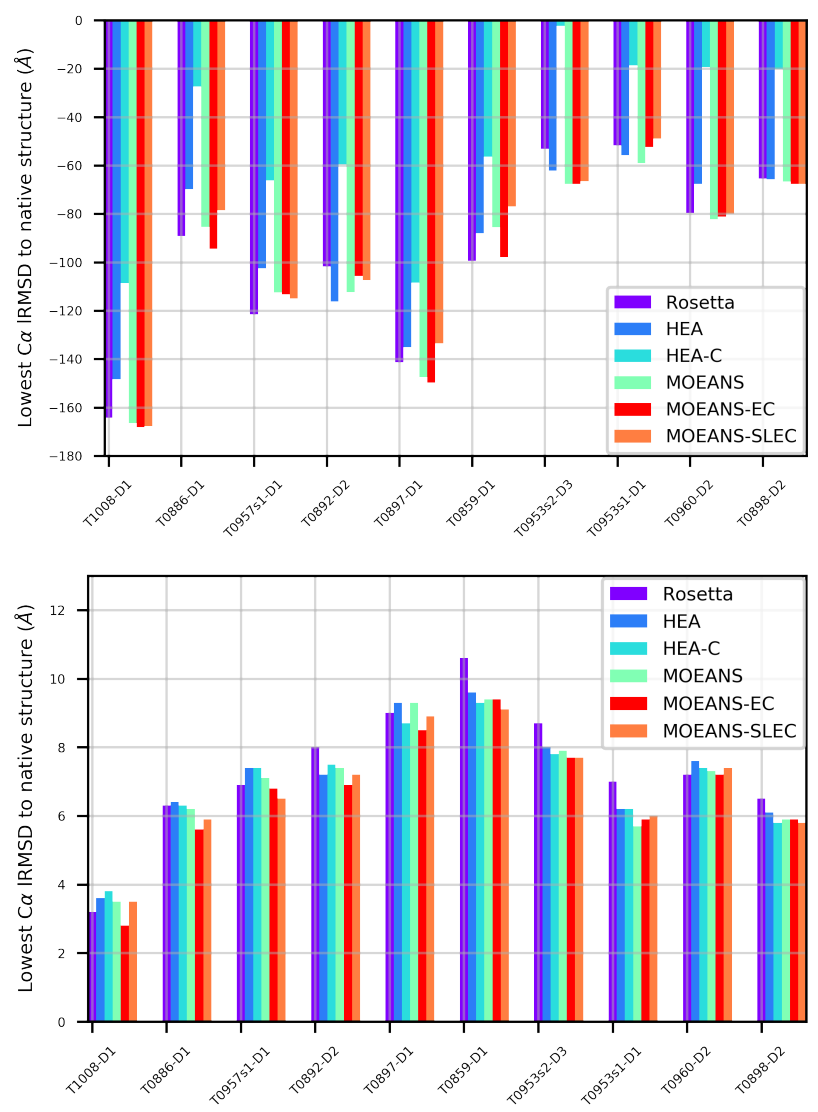

Figure 2: Comparison of (top panel) the lowest score4 (in Rosetta Energy Units - REUs) and (bottom panel) the lowest IRMSD (measured in $\AA$ ) obtained by each algorithm on each of the 10 CASP targets.

Fig. 2 compares all algorithms in terms of lowest IRMSD to the native structure. As in the benchmark dataset, HEA-C achieves higher IRMSD than all other algorithms except for HEA, where it wins on $8 / 10$ targets. This result confirms the utility of contactbased scoring in guiding towards near-native regions of the structure space. MOEANS-SLEC reaches lower IRMSD than (and so wins over) Rosetta in 8/10 targets, wins over MOEANS in 8/10 targets, over HEA in 10/10 targets, and over HEA-C in 9/10 targets. Table $1(\mathrm{~d})$ confirms the improvements by MOEANS-SLEC are statistically significant. This result also agrees with the benchmark dataset, and similar observations can be made regarding the utility of considering energetic and contact-based scoring objectives. On the other hand, MOEANS-EC beats all other algorithms in reaching lowest $1 R M S D$. It wins over Rosetta in 10/10 targets, over MOEANS in 9/10 targets, over HEA in 10/10 targets, over HEA-C in 8/10 targets, and over MOEANS-SLEC in 7/10 targets. Table 2(d) confirms these improvements by MOEANS-EC are statistically significant over all algorithms except for MOEANS-SLEC. These observations are very similar to those drawn from the evaluation on the benchmark dataset. They confirm that additional guidance by contact-based scoring in a multi-objective setting improves proximity to the native structure, but that adding too many objectives results in diminishing returns.

The comparison on GDT_TS in Fig. 3 shows similar results. HEAC outperforms only HEA on GDT_TS. MOEANS-SLEC outperforms Rosetta in 7/10 targets, MOEANS in 7/10 targets, HEA in 9/10 targets, and HEA-C in 9/10 targets. Table 1(e) confirms the improvements by MOEANS-SLEC are statistically significant over HEA and HEA-C. MOEANS-EC beats all algorithms. It outperforms Rosetta in 8/10 targets, MOEANS in 7/10 targets, HEA in 10/10 targets, HEA-C in 10/10 targets, and MOEANS-SLEC in 9/10 targets. Table 2(e) confirms these improvements by MOEANS-EC are statistically significant over all but MOEANS. These results harden our observation that guidance by both energy and contact-based scoring in a multi-objective optimization setting improves proximity to the native structure; however, adding too many objectives results in diminishing returns.

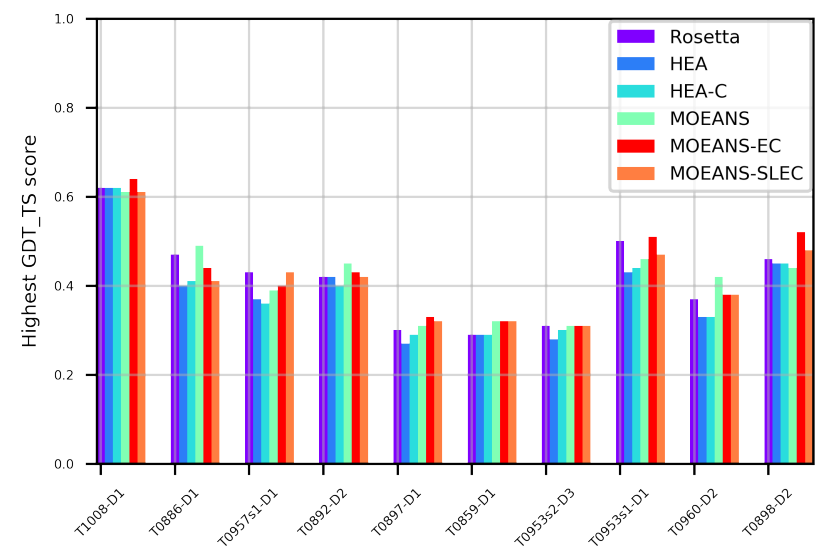

Figure 3: Comparison of the highest GDT_TS obtained by each algorithm on each CASP target.

\section{CONCLUSION}

In this paper, we investigate the role of energy and contact information in template-free PSP. The results suggest strong merit in using contact information jointly with interatomic energy in a multi-objective optimization setting. If the goal is to reach deeper in the energy surface (which evaluates exploration capability), a multi-objective optimization setting outperforms a single-objective one, unless too many optimization objectives are considered. Both observations have been drawn before by our work and work of others in PSP and hard optimization problems beyond protein modeling $[6,10,18,22]$. Higher exploration capability does not necessarily 
Table 1: Comparison of MOEANS-SLEC to other algorithms via 1-sided Fisher's and Barnard's tests. The tests evaluate the null hypothesis that MOEANS-SLEC does not achieve (a) lower lowest energy on benchmark dataset, (b) lower lowest IRMSD on benchmark dataset, (c) lower lowest energy on CASP dataset, (d) lower lowest IRMSD on CASP dataset, (e) higher highest GDT_TS on CASP dataset in comparison to a particular algorithm, considering each of the other algorithms in turn. P-values less than 0.05 are marked in bold.

\begin{tabular}{|c|c|c|c|c|c|c|c|}
\hline \multirow{3}{*}{ (a) } & Test & Rosetta & MOEANS & HEA & HEA-C & MOEANS-EC & SCDE \\
\hline & Fisher's & 0.001924 & $\mathrm{~N} / \mathrm{A}$ & 0.01282 & $7.254 \mathrm{e}-12$ & $\mathrm{~N} / \mathrm{A}$ & $\mathrm{N} / \mathrm{A}$ \\
\hline & Barnard's & 0.001118 & $\mathrm{~N} / \mathrm{A}$ & 0.008299 & $9.095 e-13$ & $\mathrm{~N} / \mathrm{A}$ & $\mathrm{N} / \mathrm{A}$ \\
\hline \multirow{3}{*}{ (b) } & Test & Rosetta & MOEANS & HEA & HEA-C & MOEANS-EC & SCDE \\
\hline & Fisher's & 0.1025 & 0.05548 & $7.254 \mathrm{e}-12$ & $2.91 \mathrm{e}-08$ & $\mathrm{~N} / \mathrm{A}$ & 0.3576 \\
\hline & Barnard's & 0.07693 & 0.03517 & $9.095 \mathrm{e}-13$ & $9.733 e-09$ & $\mathrm{~N} / \mathrm{A}$ & 0.2923 \\
\hline \multirow{3}{*}{ (c) } & Test & Rosetta & "MOEANS & "HEA & HEA-C & "MOEANS-EC & SCDE \\
\hline & Fisher's & 0.6719 & N/A & 0.3281 & $5.413 \mathrm{e}-06$ & $\mathrm{~N} / \mathrm{A}$ & N/A \\
\hline & Barnard's & 0.9991 & $\mathrm{~N} / \mathrm{A}$ & 0.2617 & $9.537 \mathrm{e}-07$ & N/A & N/A \\
\hline \multirow{3}{*}{ (d) } & "Test & Rosetta & MOEANS & " HEA & " HEA-C & MOEANS-EC & SCDE \\
\hline & Fisher's & 0.01151 & 0.03489 & $5.954 \mathrm{e}-05$ & 0.009883 & $\mathrm{~N} / \mathrm{A}$ & $\mathrm{N} / \mathrm{A}$ \\
\hline & Barnard's & 0.005909 & 0.02069 & $2.003 \mathrm{e}-05$ & 0.003978 & $\mathrm{~N} / \mathrm{A}$ & $\mathrm{N} / \mathrm{A}$ \\
\hline \multirow{3}{*}{ (e) } & Test & Rosetta & MOEANS & HEA & HEA-C & MOEANS-EC & SCDE \\
\hline & Fisher's & 0.5 & 0.5 & 0.002739 & 0.002739 & $\mathrm{~N} / \mathrm{A}$ & $\mathrm{N} / \mathrm{A}$ \\
\hline & Barnard's & 0.3883 & 0.3883 & 0.001288 & 0.001288 & $\mathrm{~N} / \mathrm{A}$ & $\mathrm{N} / \mathrm{A}$ \\
\hline
\end{tabular}

Table 2: Comparison of MOEANS-EC to other algorithms via 1-sided Fisher's and Barnard's tests. The tests evaluate the null hypothesis that MOEANS-EC does not achieve (a) lower lowest energy on benchmark dataset, (b) lower lowest lRMSD on benchmark dataset, (c) lower lowest energy on CASP dataset, (d) lower lowest IRMSD on CASP dataset, (e) higher highest GDT_TS on CASP dataset in comparison to a particular algorithm, considering each of the other algorithms in turn. P-values less than 0.05 are marked in bold.

\begin{tabular}{|c|c|c|c|c|c|c|c|}
\hline \multirow{3}{*}{ (a) } & Test & Rosetta & MOEANS & HEA & HEA-C & MOEANS-SLEC & SCDE \\
\hline & Fisher's & 0.001924 & 0.6238 & 0.01282 & $7.254 \mathrm{e}-12$ & $9.693 e-06$ & $\mathrm{~N} / \mathrm{A}$ \\
\hline & Barnard's & 0.001118 & 0.9982 & 0.008299 & $9.095 \mathrm{e}-13$ & $4.182 \mathrm{e}-06$ & N/A \\
\hline \multirow{4}{*}{ (b) } & Test & Rosetta & "MOEANS & HEA & HEA-C & MOEANS-SLEC & SCDE \\
\hline & Fisher's & 0.02808 & 0.0006159 & $3.43 \mathrm{e}-05$ & $1.523 \mathrm{e}-10$ & 0.01242 & 0.233 \\
\hline & Barnard's & 0.01924 & 0.0003401 & $1.109 \mathrm{e}-05$ & $3.729 \mathrm{e}-11$ & 0.006934 & 0.1808 \\
\hline & Test & Rosetta & MOEANS & HEA & HEA-C & MOEANS-SLEC & SCDE \\
\hline \multirow[t]{3}{*}{ (c) } & Fisher's & 0.01151 & 0.08945 & 0.01151 & $5.413 e-06$ & 0.03489 & $\mathrm{~N} / \mathrm{A}$ \\
\hline & Barnard's & 0.005909 & 0.05789 & 0.005909 & $9.537 \mathrm{e}-07$ & 0.02069 & N/A \\
\hline & Test & Rosetta & MOEANS & HEA & HEA-C & MOEANS-SLEC & SCDE \\
\hline \multirow[t]{3}{*}{ (d) } & Fisher's & $5.954 \mathrm{e}-05$ & 0.009883 & $5.413 \mathrm{e}-06$ & 0.01151 & 0.1849 & $\mathrm{~N} / \mathrm{A}$ \\
\hline & Barnard's & $2.003 e-05$ & 0.003978 & $9.537 \mathrm{e}-07$ & 0.005909 & 0.1317 & $\mathrm{~N} / \mathrm{A}$ \\
\hline & Test & Rosetta & "MOEANS & HEA & "HEA-C & "MOEANS-SLEC & $\overline{\mathrm{SCDE}}$ \\
\hline \multirow[t]{2}{*}{ (e) } & Fisher's & 0.03489 & 0.325 & $5.413 e-06$ & $5.413 e-06$ & 0.02864 & $\mathrm{~N} / \mathrm{A}$ \\
\hline & Barnard's & 0.02069 & 0.2617 & $9.537 \mathrm{e}-07$ & $9.537 \mathrm{e}-07$ & 0.01139 & N/A \\
\hline
\end{tabular}

translate to better proximity to the native structure. Our evaluation clearly makes this case. Considering a contact-based scoring in the selection operator, whether in isolation in a single-objective multi-optimization setting (as in HEA-C) or jointly with energy in a multi-objective optimization setting (as in MOEANS-EC and MOEANS-SLEC), improves proximity to the native structure. Taken altogether, considering energy and contact-based scoring jointly, as separate optimization objectives (but not too many objectives), performs best in both reaching lower energies and better proximity to known native structures. While we focus on an evolutionary algorithmic framework here for the ease with which it allows investigating different strategies, other algorithmic frameworks can be considered. We hope the presented study will encourage researchers to further investigate combinations of different structure evaluator functions for advancing template-free PSP.

\section{ACKNOWLEDGMENTS}

This work is supported in part by NSF IIS Grant No. 1763233. Computations were run on ARGO, a research computing cluster provided by the Office of Research Computing at George Mason University, VA (URL: http://orc.gmu.edu). 


\section{REFERENCES}

[1] B. Adhikhari, J. Hou, and J. Cheng. 2018. DNCON2: improved protein contact prediction using two-level deep convolutional neural networks. Bioinformatics 34, 9 (2018), 1466-1472.

[2] Christian B. Anfinsen. 1973. Principles that Govern the Folding of Protein Chains Science 181, 4096 (1973), 223-230.

[3] G. A. Barnard. 1945. A new test of 2x2 tables. Nature 156 (1945), 177.

[4] H. M. Berman, K. Henrick, and H. Nakamura. 2003. Announcing the worldwide Protein Data Bank. Nature Structural Biology 10, 12 (2003), 980-980.

[5] N. J. Cheung and W. Yu. 2018. De novo protein structure prediction using ultrafast molecular dynamics simulation. PLOS ONE 13 (11 2018), 1-17.

[6] K. A. De Jong. 2006. Evolutionary Computation: a Unified Approach. MIT Press, Cambridge, MA.

[7] R. Evans, J. Jumper, J. Kirkpatrick, L. Sifre, T. F. G. Green, C. Qin, A. Zidek, A. Nelson, A. Bridgland, H. Penedones, S. Petersen, K. Simonyan, S. Crossan, D. T. Jones, D. Silver, Kavukcuoglu K., D. Hassabis, and A. W. Senior. 2018. De novo structure prediction with deep-learning based scoring. Thirteenth Critical Assessment of Techniques for Protein Structure Prediction (Abstracts) (2018).

[8] R. A. Fisher. 1922. On the interpretation of $\chi^{2}$ from contingency tables, and the calculation of P. 7 Roy Stat Soc 85, 1 (1922), 87-94.

[9] M. Gao, H. Zhou, and J. Skolnick. [n. d.]. DESTINI: A deep-learning approach to contact-driven protein structure prediction. 9, 3514 ([n. d.])

[10] J. Handle, D. B. Kell, and J. Knowles. 2007. Multiobjective optimization in bioinformatics and computational biology. IEEE/ACM Trans Comput Biol and Bioinf 4 2 (2007), 279-292.

[11] S. M. Kandathill, M. Garza-Fabre, J. Handle, and S. C. Lovell. 2018. Improved fragment-based protein structure prediction by redesign of search heuristics. Scientific Reports 8 (2018), 13694.

[12] A. Liwo, J. Lee, D. R. Ripoll, J. P, and H. A. Scheraga. 1999. Protein structure prediction by global optimization of a potential energy function. Proc Natl Acad Sci USA 96, 10 (1999), 5482-5485.

[13] A. D. McLachlan. 1972. A mathematical procedure for superimposing atomic coordinates of proteins. Acta Cryst A 26, 6 (1972), 656-657.
[14] Jens Meiler and David Baker. 2003. Coupled prediction of protein secondary and tertiary structure. Proc Natl Acad Sci USA 100, 21 (2003), 12105-12110.

[15] R. Nussinov and P. G. Wolynes. 2014. A second molecular biology revolution? The energy landscapes of biomolecular function. Phys Chem Chem Phys 16, 14 (2014), 6321-6322.

[16] B. Olson, K. A. De Jong, and A. Shehu. 2013. Off-Lattice Protein Structure Prediction with Homologous Crossover. In Conf on Genetic and Evolutionary Computation (GECCO). ACM, New York, NY, 287-294.

[17] B. Olson and A. Shehu. 2013. Multi-Objective Stochastic Search for Sampling Local Minima in the Protein Energy Surface. In ACM Conf on Bioinf and Comp Biol (BCB). Washington, D. C., 430-439.

[18] B. Olson and A. Shehu. 2014. Multi-Objective Optimization Techniques for Conformational Sampling in Template-Free Protein Structure Prediction. In Intl Conf on Bioinf and Comp Biol (BICoB). Las Vegas, NV, 143-148.

[19] J. Schaarschmidt, B. Monastyrskyy, A. Kryshtafovych, and A. Bonvin. 2018. Assessment of contact predictions in CASP12: Co-evolution and deep learning coming of age. Proteins 86, Suppl 1 (2018), 51-66.

[20] A. Shmygelska and M. Levitt. 2009. Generalized ensemble methods for de novo structure prediction. 106, 5 (2009), 94305-95126.

[21] Sheng Wang, Siqi Sun, Zhen Li, Renyu Zhang, and Jinbo Xu. 2017. Accurate De Novo Prediction of Protein Contact Map by Ultra-Deep Learning Model. PLOS Computational Biology 13, 1 (01 2017), 1-34.

[22] A. Zaman and A. Shehu. 2019. Balancing multiple objectives in conformation sampling to control decoy diversity in template-free protein structure prediction. BMC Bioinformatics 20, 1 (2019), 211. https://doi.org/10.1186/s12859-019-2794-5

[23] A. Zemla, C. Venclovas, J. Moult, and K. Fidelis. 2001. Processing and evaluation of predictions in CASP4. 45 (2001), 13-21.

[24] C. Zhang, S. M. Mortuza, B. He, Y. Wang, and Y. Zhang. 2018. Template-based and free modeling of I-TASSER and QUARK pipelines using predicted contact maps in CASP12. Proteins: Struct, Funct, and Bioinf 86, S1 (2018), 136-151.

[25] G. Zhang, L. Ma, X. Wang, and X. Zhou. 2018. Secondary Structure and Contact Guided Differential Evolution for Protein Structure Prediction. IEEE/ACM Trans Comput Biol and Bioinf (2018), 1-1. 